\title{
Threshold Alarm Algorithm for in-Patient Monitoring System
}

\author{
Pushpa $\mathrm{G}^{1}$, Rachana B S ${ }^{2}$ \\ PES University
}

\begin{abstract}
As the Machinery Science is advancing and data is growing in medical domain, requires vital increasing demands for rapid changes in supervising patients in hospital, as well as for observing in-patients in hospital. CCTv's, sensors and devices connected to Wi-fi may collect the patient daily routine activities for entire day. The sensors gather and exhibits patient's raw conditions which enables the detector system catching the validated information to avoid life-threatening situations of patients. In this work we present a Threshold Alarm Algorithm to suppress false alarms rates and number of alarms for specific purpose for critical situations for In-patient supervising system which can be set up in the hospital special ward, where the present system uses physical system and application level behavioral information conditions along with previous health details of the related patients. This proposed has been tested by considering a five special wards of fifteen in number with 3 patients in each ward along variant diseases and behavioral conditions. The outcomes are quite inspiring and it has been error tolerant up to $85 \%$ by reducing false alarm rates and number of alarms by deploying generic super alarm for multipurpose.
\end{abstract}

Keywords: Wi-fi related sensors, conditional parameters, cellular networks, patient monitoring, routing algorithms, alarm fatigue in patient monitors system.

\section{I.INTRODUCTION}

As great amount of low-performance alarms drains the required goal of monitoring patient in critical scenarios becomes first priority to observe and recognize patient behavioral patterns. With regard to the change in current systematic overview and reviews of patient alarm fatigue and therefore the interventions addresses the matter of inconsistent data, reduction in number of low-rated alarms and improving alarm quality by best results extracted from it's been the foremost goal-therefore the interventions often squeezed the present system without fundamentally disrupting the core of patient monitoring methodology - the embedded signal processing and sensor specific algorithms that extracts the assorted patterns for alarm oriented instances of patient behavior. Given the rapid adoption of electronic health record (EHR) systems and continuous growth of knowledge science and analytics, the opportunities and potentially transforming success rates are within the reach to undertake the danger of improving and enhancing the performance of core patient monitoring algorithms by taking advanced features of an ever growing and increasingly connected technological IT system in health care enterprise applications. By involving the keen surfing of fundamental algorithms, not only the low-performance alarms are reduced but also number in alarms are going to be reduced by considering more predictive monitoring patterns of current patient state are going to be achieved to enable accurate results. The alarm annotations in real time at the bedside indicate that about $80 \%$ of those alarms were clinically assessed and relevant true positives; $8 \%$ were true positives without clinical relevance; and seven were false positives. These results uses as an improved specificity of the alarm algorithms within the way forward for bedside monitoring systems and demonstrates that the designed real time-data acquisition system enables prediction and evaluation of patient monitoring algorithms for critical clinical care applications.

In proposed system, we consider various key elements and scenarios for implementing algorithm for suppressing existing false, inactive alarms and to deal with lack of precision in critical care of patient health data. In recent years, in step with a recent systematic review of patient monitor alarm fatigue and therefore the interventions to handle the matter, 2 reduction of the amount of alarms rather than improving alarm quality has been the first targettherefore the intervention often tweaked an existing system without fundamentally transforming the core of patient monitoring technology.

Given the rapid adoption of electronic health record (EHR) systems and advancement of information science, the opportunities and potentially transforming success are within the reach to undertake the challenge of improving core patient monitoring algorithms by taking advantage of an ever increasingly connected technological ecosystem in an exceedingly healthcare enterprise. By pursuing fundamental algorithm research, not only the matter of alarm fatigue are going to be solved but also a more predictive monitoring of patient state are going to be achieved to enable proactive interventions. The alarms of treatment are the first concern of critical care and want. The frequent false alarms not only are a nuisance for patients and caregivers but may compromise patient safety and effectiveness of care. the event of alarm systems has lagged behind the technological advances of medical devices over the last 25 years. From a clinical perspective, major improvements of alarm algorithms are urgently needed. this provides an outline of current scenarios of clinical and medical systems and also the underlying problems that susceptible to the discussion on different methods from statistics and computational science and their potential for growth and enhancements in clinical application $[1,2]$.

Our proposed algorithm involves reduction of false alarms, all life-threatening situations shall be detected and alarmed, regardless of whether or not they are patient- or devicerelated. Patient specific alarm are distinguished from device 
specific algorithms to cut back false alarms and number of poor-quality alarms.

\section{PATIENT RELATED ALARM ALGORITHMS}

Alarms are typically displayed in two forms: 1. One with Acoustic form -where the alarm is given as a warning sound. Most manufacturers distinguish the priority of an alarm with different signals. Most alarms have intuitive variations in tones (e. g. 'short-long-short' for 'ventilate) have been the object of research but have not found their way into routine clinical practice. Alarms directly project the organ systems, device hardware, or parts of it (e. g., ventilation or circulation) or alarms with direct mentioning of the physiological problem ('blood pressure' or 'pulse rate') have also not been introduced into practice. 2. Visual screen alarms involve mostly flashing or coloring of the related context parameter in an eye-catching manner. Some systems provide integrated displays of several parameters. One example is a spider-display, which shows the relationship of different parameters in a stylized spider web. Such applications can be useful to display different parameters in context including behavioral parameters. Compared to other professions in industry and aviation, adoption of such new displays in healthcare has been slow and stayed backward. Alarm-related problems help to prevent patient critical situations by providing rapid recognition of reaction to critical situations, but only if they are not 'false alarms. Medical progress leads to an increasing number of 'monitorable' parameters and thus an increasing number of possible alarms [2.3].

\section{Equipment related algorithms: Smart alarms for medical devices}

According to the survey on Smart Alarms techniques exhibits algorithm methods that considers multi-instance variables for critical signs when monitoring critical situation of patient reacted to heart surgery. The results of this gives the benefit for algorithm to focus on monitoring four major signs: blood pressure, heart rate, respiration rate, and oxygen saturation rate. The Smart alarm algorithm creates a new, three level alarm priority system that gathers information accurately. Preferences for visual and System Validation Penn Presbyterian Medical Centre (PMC)The Smart Alarm Manager was tested using retrospective vital sign and contextual data from de-identified PMC SICU patients. The data was obtained with expedited IRB approval of Protocol \#811401.

Physio Net: The system was also tested with clinical data provided by PhysioNet.org, an MIT databank.

Results from Physio Net show:

- Number of alarms decreased by $59 \%(\mathrm{n}=6737)$.

- Quiet time increased by $101 \%(\mathrm{n}=421 \mathrm{hrs})$.

Results from PMC show:

- Number of alarms reduced by $52 \%(\mathrm{n}=51)$.

- False positives reduced by $56 \%(\mathrm{n}=52)$.

- No change in false negatives, or missed alarms.
With a multivariate expert system, the Smart Alarm model succeeded in decreasing the number of false alarms and poor-quality alarms for structured patient data $[3,4,5]$.

\section{PROPOSED THRESHOLD ALARM ALGORITHM FOR INPATIENT ALGORITHM}

The proposed algorithm consists of two components 1.Mtehodological approach for patient context information 2.Artificial intelligence approach for predicting the status of patient based on daily activities.

The proposed alarm algorithm is the combination of both methodological and artificial approach where methodological approach involves step by step procedure to optimize the patient context information by considering only behavioral and functional parameters combination of patient in hospital setting whose staff deals with alarm notification daily basis.

The Artificial intelligence system (decision support system used to predict the status of patient by considering important vital signs variations by comparison to the normal threshold levels.

1.To overcome the technically false alarms by considering appropriate patient data (ignoring missed values or reconstruct) and fed into database acquisition system without suppressing the threshold level of alarm.

2. The proposed algorithm is the combination of both vital and visual signs for monitoring patient in alarm in both modes I.e. sound and visual screening effect.

3.The algorithm works on accurate patient data by ignoring inappropriate behavior information of patients.

4.The Super algorithm is the combination of both univariate i.e. (by improving the detection patterns of change in a specific variable and multivariate (simultaneous analysis of more than one variable) based on the behavior and situation of patient.

5.The proposed algorithm gives warn sound before life threatening situations occurs and physiologic /diagnostic information with reference to current situation of patient. 6.The proposed algorithm results in reduction false alarm rates up to $90 \%$ and reduction in number of alarms used for specific purpose for unstructured type of patient data by considering combination of unit and multivariate parameters based on situation (ignoring missed data).

\section{THE ARCHITECTURE OF PROPOSED ALGORITHM:}

The architecture of proposed system has two components:

1. Patient context information module

2. Patient specific alarm module. 


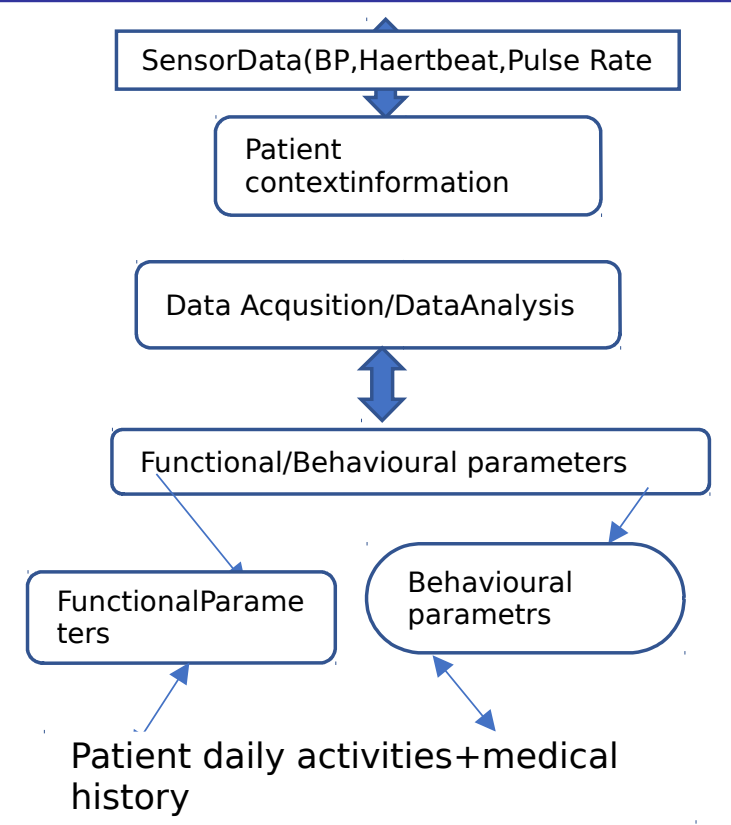

Fig1: Patient context information module

A:CI Acquisition Module: The CI Acquision section includes general section of patients with wireless sensors. The sensor captures the vital activities of the patients and the context information that includes the physical activities and the behavioral activities of the patients.

The context information acquisition module captures the physical context information, function-based context and behavior-based context information of the patient.

All the context parameters of the patient are acquired by the Context information acquisition sub system.

B: CI Analysis Module: By using the gathered information from CI module, context information activity templatebased database, $\mathrm{CI}$ analysis module will analyze and extracts the current patient activity and it reconstruct or ignore the missing context attributes by assigning it to Null or removing it. Context information activity database consists of daily activities of the patients such as whether the patient is walking, sleeping, shouting in the room, taking food or medicine and so on. The patient history database consists of all the information of the patient such as name, age, address, disease, surgeon/doctor who is treating that patient, date of admission, tests conducted, reports produced, medicine prescribed and patient's previous medical history information.

The context information pertaining to the patient collected from the CI module will be analyzed by the context information analysis system. Behavioral, physical parameters and vital signs of patient are gathered by sensor nodes which are deployed in the ward. The captured context parameters from the CI module will be match with the activity template-based database to predict the current activity of the patient. The system computes the certainty factor based on the number of parameters going to match with the different activities against the total number of the input. Based on the value of the certainty factor, the system will predict the current activity of a patient. Generally, the certainty factor ranges from 0 to 1 .
The input given by the user consisting of the attribute values for all the categories of context parameters behavioural and functional context parameters, which is represented by the equation 1 . The proposed system derives the activity by adding the attribute values of each of the context parameters. Equation 2 represents the activity derived by the system.

$$
\begin{aligned}
& \text { Activity }=\sum_{i=1}^{l} P C+\sum_{j=1}^{m} C F+\sum_{k=1}^{n} C B \\
& \text { Activity }=\sum_{i=1}^{l} P C+\sum_{j=1}^{m} C F+\sum_{k=1}^{n} C B
\end{aligned}
$$

Eq 2

Depending on the input given by the user the system calculates the certainty factor that ranges from 0 to 1 . If the certainty factor is 1 the system predicts the activity of a patient correctly, if it lies within the value 1 then it predicts the patient activity as a probability of ay or may not be cases. The current activity of the patient is predicted by the system intelligently and it in turn triggers the alerts/SMS to doctors, patient's care takers and to the hospital management staff based on criticality of the patient.

The patient specific alarm module shown above module has patient behavioural context information like patterns of normal and extreme behaviour of patient, I,e is identified by presently considering the vital signs like BP,Pusle rate and ECG during daily activities of patient through sensors.

The proposed patient specific alarm module has systems of predictor which will predict the behaviour of patient by considering threshold level fixed for each vital signs of monitoring.

The component of knowledge base in system has patterns of normal and abnormal behaviour of patient including past history for the predicting the state of patient.

The detector behaviour will detect the patient status of life in danger or not if any of threshold level of vital signs exceeds through alarm triggering.(Threshold for BP is $120 / 80,82$ for Pulse rate,80 for ECG in general).The anomalous frequency agents have threshold level of each vital signs of patient recorded through sensors[5,6].

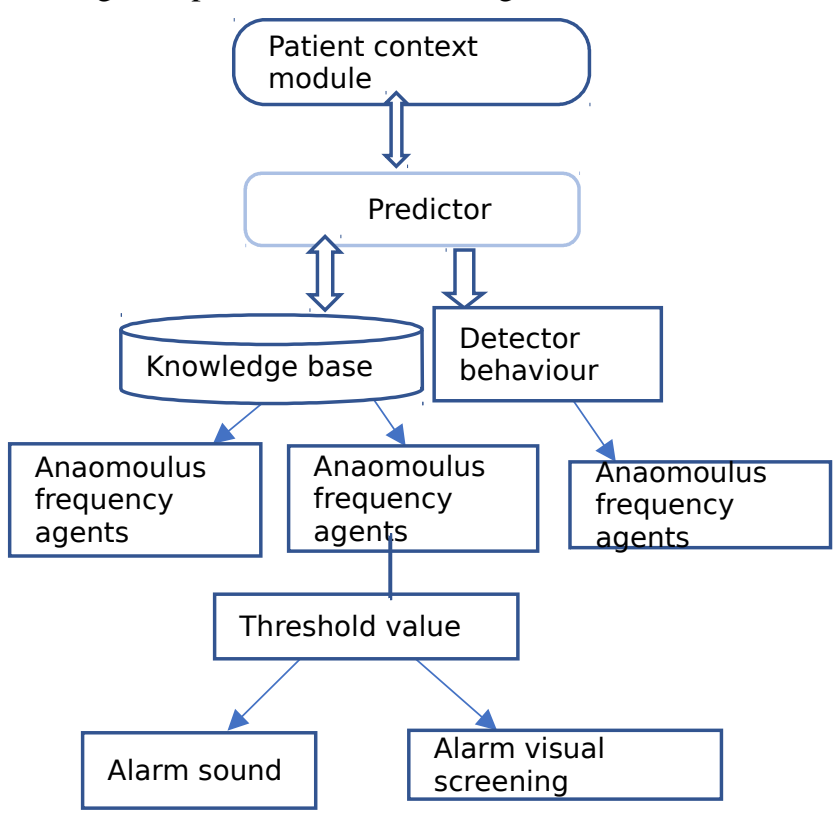

Fig2: Patient specific threshold alarm module 


\section{SIMULATION RESULTS AND SNAPSHOTS Table}

4.1 Behavioral context parameters sample for alarm

\begin{tabular}{|c|c|c|c|c|c|}
\hline of & $\begin{array}{l}\text { Patient daily } \\
\text { activity }\end{array}$ & $\begin{array}{c}\text { Health } \\
\text { parameters } \\
\text { :BP,Pulsera } \\
\text { te,ECG }\end{array}$ & Year & $\begin{array}{l}\text { Thresh } \\
\text { old }\end{array}$ & results \\
\hline & Sleeping & $120,70,75$ & 2010 & 130,90 & normal, no alarm \\
\hline & shouting & $140,100,89$ & 2010 & 130,90 & $\begin{array}{l}\text { abnormal ,trigger } \\
\text { sound alarm }\end{array}$ \\
\hline & Resteless & $140,100,90$ & 2010 & 130,90 & $\begin{array}{l}\text { abnormal ,trigger } \\
\text { sound alarm }\end{array}$ \\
\hline 1 & Not taking food & $155,100,91$ & 2010 & 130,90 & $\begin{array}{l}\text { abnormal ,trigger } \\
\text { sound alarm with }\end{array}$ \\
\hline & Not breathing & $180,100,92$ & 2010 & 130,90 & $\begin{array}{l}\text { abnormal, trigger } \\
\text { sound alarm with }\end{array}$ \\
\hline 2 & Sleeping & $120,70,75$ & 2010 & 130,90 & normal ,no alarm \\
\hline & Breathing & $120,70,76$ & 2010 & 130,90 & normal, no alarm \\
\hline & Medicine taken & $120,70,80$ & 2010 & 130,90 & normal ,no alarm \\
\hline 2 & Taking food & $120,70,82$ & 2010 & 130,90 & normal ,no alarm \\
\hline & \begin{tabular}{|l} 
medicine not \\
taken
\end{tabular} & $120,70,83$ & 2010 & 130,90 & normal, no alarm \\
\hline & tidiness & $120,70,84$ & 2010 & 130,90 & alrm sound \\
\hline 3 & Anxiety & $120,70,85$ & 2010 & 130,90 & alrm sound \\
\hline & Not taking food & $120,70,86$ & 2012 & 130,90 & alrm sound \\
\hline 4 & Breathing & $120,70,80$ & 2013 & 130,90 & normal ,no alarm \\
\hline 4 & Walking & $110,70,80$ & 2014 & 130,90 & normal, no alarm \\
\hline & Taking food & $120,82,80$ & 2015 & 130,90 & normal ,no alarm \\
\hline 4 & Takinng Medicin & $120,82,81$ & 2016 & 130,90 & normal, no alarm \\
\hline 4 & Not breathing & $120,82,82$ & 2017 & 130,90 & normal ,no alarm \\
\hline 4 & Sleeping & $120,82,83$ & 2010 & 130,90 & normal, no alarm \\
\hline & Tidiness & $120,82,84$ & 2010 & 130,90 & normal, no alarm \\
\hline & Restless & $120,82,85$ & 2011 & 130,90 & normal ,no alarm \\
\hline & Restless & $120,82,86$ & 2012 & 130,90 & normal ,no alarm \\
\hline & food taken & $120,82,87$ & 2012 & 130,90 & normal , no alarm \\
\hline & medicine taken & $129,82,88$ & 2012 & 130,90 & normal, no alarm \\
\hline & not sleeping & $129,82,89$ & 2012 & 130,90 & normal ,no alarm \\
\hline & walking & $129,82,90$ & 2012 & 130,90 & abnormal ,aalrm \\
\hline & food taken & $129,82,91$ & 2012 & 130,90 & abnormal , aalrm \\
\hline & walking & $129,82,92$ & 2012 & 130,90 & abnormal , aalrm \\
\hline & walking & $129,82,93$ & 2012 & 130,90 & abnormal ,aalrm \\
\hline & walking & $129,82,94$ & 2012 & 130,90 & abnormal , aalrm \\
\hline & walking & $129,82,95$ & 2012 & 130,90 & abnormal ,aalrm \\
\hline
\end{tabular}

The above table 4.1 records simulation of sample of 5 patients in two special wards where reduce the false alarm rates by ignoring inconsistent data and only collecting behavioural data for prediction of life-threatening condition of patient in hospital. The patient specific alarm will be working as both device specific and patient specific by considering multivariate context parameters of patient monitoring system,by considering $\mathrm{N}$ patients rooms has 3 beds and 6 pairs of lights, 4 fans and each bed side there are two small tools to keep food and medicine for the patient. This room is equipped with specific pressure, temperature activity-based sensors along with some CCTVs[4][5][6]. The equipment will provide more than 100 context-based attributes for screening the activities of the patients on daily basis. The simulation process is done for 5 daily activities having behavioral parameters used every patient in current location of status to predict normal situation to trigger visual alarm or life-threatening situation to beep alarm as shown in the graphs 1,2 and 3 below and table 4.1 above.

In the simulation process system will periodically collects the information from the context information module and maintains the patient activity by examine the given context information with the standard context-based database, which leads to identify the activities of each patient. Depending on the type of activity, the system advices medical staff likes nurse, doctor and ward boy as per the requirement. The graph 1 shows the results for shouting activity by plotting the graph for the number of vital attribute matches against threshold level of behavioral context information, times that attributes appeared[5][6].

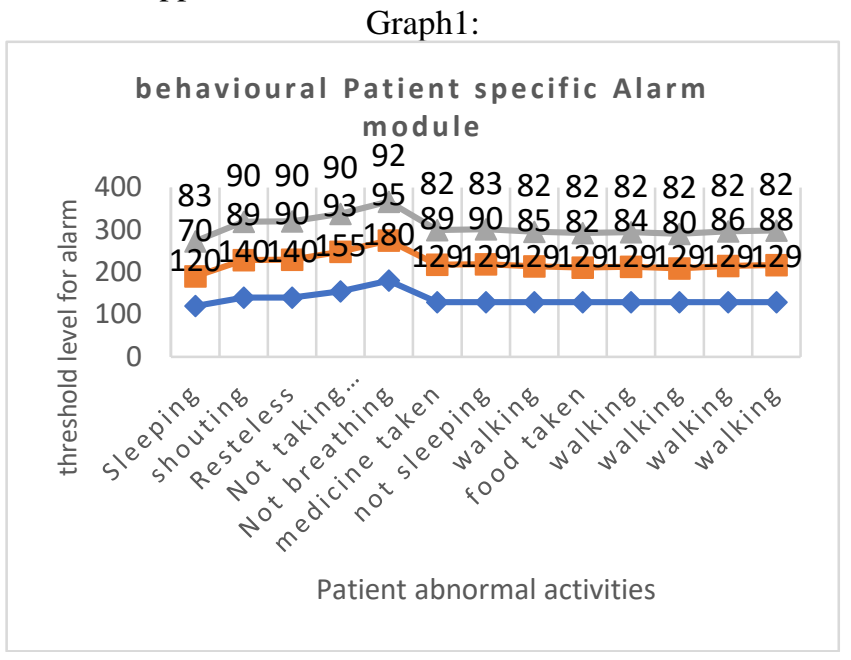

Graph 2:

\section{Behavioural Parameters}

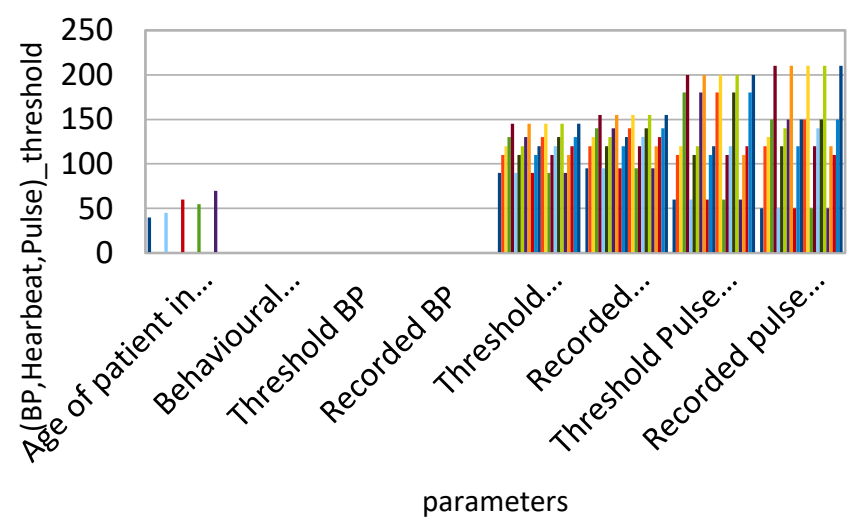

Graph 1: The following graph1 shows simulation results for 15 patients in 5 special wards three patients in each with super alarm for vital signs like BP, Pulse rate and ECG rate in corresponding year and results based on behavioral context information is achieved.

The threshold value fixed for the normal patient for BP, Pulse rate and ECG rate is $120 / 80,60-100,60-82 \mathrm{bpm}$. If anyone varies among three alarm triggers with message, otherwise, if majority factors vary alarm sound will be issued and if all varies alarm sound with visual screening will be issued.

Graph2: The graph2 shows the accuracy of the parameters taken for different trails for the "patient is abnormal" activity behavioral context information module to trigger alarm by simulating 15 patients in 5 special wards by considering variation in threshold levels of vital signs of patient's daily activities.[4][5] 


\section{CASE STUDY}

1. Considering 15 patients in five special wards for Behavioral context parameters in hospital ward for alarm trigger module:

Recognition of different ways of shouting in a ward in the hospital is done by creating "shouting" template with below context parameters.

$>$ Variation in voice tone (low or high)

$>$ Extreme sound in ward location

$>$ High range in voice tone due to anger/pain/anxiety

$>$ Proper medicine or food not taken

$>$ Tensed expression and extreme body movements. Parameters to recognize only the doctor is speaking with high pitch voice:

$>$ High variation in voice

$>$ Doctor movements to have control and confront patient

Parameters only to recognize employees to confront and control patients

$>$ High variation in voice

$>$ Employees_hand_movements for controlling and confront patients.

In all these ways of variation one behavior parameter shouting is considered by matching the threshold levels of vital signs of patient to predict normal or life-threatening condition of a patient by feeding the relevant information to patient specific super alarm module to trigger alarm through message or sound appropriately.

\section{CONCLUSION}

Monitoring the patient activity in the Private clinic or Nursing homes is a complex task. In order to reduce the false rate and number of alarms in monitoring a patient, our proposed system runs the algorithm that collects the behavioral context information, analyze and intelligently predict the current activity of a patient by comparing the threshold of vial signs of patient The system triggers the alarm with warning sound to intimate the doctor, nurse and hospital management staff if it detects the life threatening situation of the patient otherwise just gives the visual screen as a caution to take care of patient behavior that leads to normal state.

\section{REFERENCES}

[1] By Eun-Shim Nahm, $\mathrm{PhD}, \mathrm{RN}$, "Innovations in patient- monitoring systems: Wireless devices and other advancements help clinicians make better treatment decisions".

[2] Tita Alissa Bach, Lars-Martin Berglund, Eva Turk," Mangaing alarm systems for quality and safety within the hospital setting ",2018.

[3] Michael Imhoff, MD, PhD*, and Silvia Kuhls, "Alarm Algorithms in Critical Care Monitoring",2006

[4] Felix Schmid*, Matthias S Goepfert, Daniel A Reuter.'Patient monitoring alarms in the ICU and in the surgery ",2013

[5] Xia huo,Monica Sap0, Val Nenov, Tod Barry,Sunghan Kim,Duch H Do,Noel Boyel,Neil Martin,'Predictive combinations of monitor alarms preceding in hospital code blue-events",2012.

[6] Charalampos Tsimenidisl, Alan Murrayl," Reliability of Clinical Alarm Detection in medical aid Units",2015.
AUTHORS PROFILE

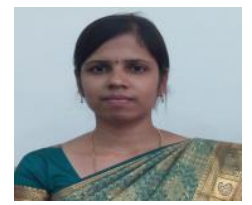

Pushpa G, MTech in CSE, Asst.Prof Department of Computer Science \&Engineering,PESIT,RR Campus,Banglore-85.

Publications: [1] Chandraprabha K S, Nagaratna B Chittaragi, Pushpa G,

Dr. Pallappa Venkataraman IISc,Bangalore,"Context

Based In-patient Monitoring System",IEEE Symposium ,2017.

[2] Rachana B S, Pushpa G," Clustering of Data using Affinity algorithm",

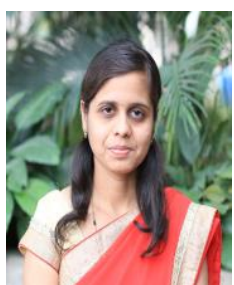

Rachana B S, MTech in CSE, Asst.Prof epartment of Computer Science \&Engineering,PESIT,RR Campus,Banglore-85.

Publications:[1] Rachana B S,Pushpa G,"Clustering of Data using Affinity algorithm",IJERT, Dec 2019. 